\title{
Una introducción a la ética pragmaticista
}

\author{
An Introduction to Pragmaticistic Ethics
}

\section{Uma introdução à ética pragmaticista}

Fecha de entrega: 5 de noviembre de 2014 Fecha de evaluación: 25 de noviembre de 2014 Fecha de aprobación: 15 de diciembre de 2014

Juliana Acosta López de Mesa*

\section{Resumen}

Se busca argumentar la concepción y el sentido de la ética en Peirce, teniendo en cuenta que es más una propuesta de su alcance que una presentación de lo que el filósofo norteamericano expuso. Peirce escribió pocas líneas sobre la función normativa de la ciencia de la ética y de su papel en la filosofía en general, de ahí la necesidad de reconstruir y complementar el vínculo de este saber teorético con la reacción entre fenómenos según unos fines de acción determinados. Para esta labor se realiza una discusión con escritos actuales de especialista que han intentado abordar y explicar la temática.

Palabras clave: pragmaticismo, ética, ciencias normativas, summum bonum.

* Filósofa de la Universidad de Antioquia, realizó sus estudios de maestría y doctorado en Southern Illinois University, investigando sobre las categorias de la libertad en Peirce, bajo la dirección de Douglas Anderson. Ha publicado artículos en revistas especializadas sobre estudios de filosofía ética, especialmente en Aristóteles. Correo: acostajuliana1@hotmail.com 


\section{Abstract}

The idea is to argue the conception and sense of Peirce's ethics, considering that it is more a proposal about its scope than about what the philosopher said. Peirce wrote just a few lines about normative ethics and its role in philosophy in general, therefore the need to rebuild and complement the link of this theoretical knowledge with the reaction between phenomena as a purpose for certain actions. To accomplish this work there will be a debate with current writings from specialists who have tried to approach and explain the theme.

Keywords: Pragmaticistic, ethics, normative sciences, summum bonum.

\section{Resumo}

Buscamos no presente artigo argumentar a concepção e o sentido da ética em Peirce, propondo uma consideração sobre o seu alcance ao invés de uma apresentação das ideias do filósofo norte-americano. Peirce escreveu poucas linhas a respeito da função normativa da ciência, da ética e de seu papel na filosofia geral, é nesse sentido que evidenciamos a necessidade de reconstruirmos e complementarmos o vínculo deste saber teórico com a reação entre fenômenos segundo fins de ação determinados. Para este trabalho, realizouse uma intertextualização entre os artigos atuais de especialistas, cuja abordagem busca explicar a temática.

Palavras-chave: Pragmatismo, ética, ciências normativas, summum bonum.

\section{Los estudios sobre la ética de Peirce en contexto}

Como el título de esta reflexión indica, la intención es hablar de la ética en Peirce a modo introductorio, esto por varias razones. Una de ellas es que el tema de por sí es, si se quiere, inédito en el ámbito de la escolaridad peirceana. Tradicionalmente, mientras existen muchos quienes afirman que no existe tal cosa como una ética peirceana, hay otro tanto que aceptan tímidamente su existencia y sólo hasta hace relativamente poco se vienen realizando trabajos sistemáticos en el tema, entre dichos 
intérpretes se encuentran cuatro autores Cheryl Misak, Edward Petry, Hedy Boero y David Pfeifer. De las personas mencionadas, Misak es la persona más reconocida a nivel mundial gracias a su libro Truth, Politics, Morality: Pragmatism and Deliberation del año (2002), pero su versión de la ética peirceana en este libro deja mucho que desear, ya que como ella misma lo reconocerá más tarde, en su libro Truth and the End of Inquiry: A Peircean Account of Truth del año 2004 "La posición cognitivista que articulo allí [en Truth, Politics, Morality] se presenta muy a favor de Peirce como una posición que él debería haber sostenido, pero no la que en realidad sostuvo" (170)

No sorprende entonces el hecho de que mucho de lo que afirma Misak en su libro del año 2002 realmente no hace justicia a la propuesta filosófica de Peirce, pues entre muchas otras cosas, padece de uno de los males que afectan a la mayoría de los académicos peirceanos al afirmar que, para Peirce, el ámbito de la ética y el de la ciencia no tienen nada en común y están totalmente separados. Aspecto, que si bien parece de menor cuantía, realmente afecta de manera profunda cualquier intento de proponer una ética en la filosofía de este autor.

Misak (2004, p. 162), se retracta de la siguiente manera:

En este capítulo muestro lo que anteriormente pensé que era imposible: el mismo Peirce, pese a algunos giros confusos en su presentación, nos presenta una versión cognitivista de la ética coherente y sofisticada —una versión en la que el juicio moral es traído bajo el ámbito de la verdad, el conocimiento y la investigación. Contrario a lo que muestran las primeras apariencias, Peirce no distingue entre ciencia y ética en razón de que la ciencia apunta a la verdad y la ética hacia una creencia rápida. Tanto la ciencia como la ética apuntan a la misma cosa: a la creencia verdadera que resiste ante la experiencia y el argumento (171-172), pero de esta retractación sólo consta el último capítulo de su libro y hasta el momento no tengo noticia de que haya elaborado desarrollos sistemáticos sobre el problema. Petry (1990), por otro lado, tiene, a mi juicio, un excelente y hermoso trabajo de tesis doctoral sobre el concepto de auto-control en Peirce, del cual se encuentra publicado solamente un artículo en la revista Transactions, que es la revista más importante en lo que a estudios peirceanos se refiere y su tesis ni siquiera se encuentra en la Universidad de Pennsylvania, a la cual pude tener acceso gracias a mi asesor, amigo de juventud del autor. Por último, encontramos a Heidy Boero, una académica argentina formada en el grupo de estudios de Navarra, quien acaba de publicar su libro Claves para 
una ética pragmaticista. En mi concepto, este es uno de los mejores trabajos sistemáticos en torno a la ética de Charles S. Peirce. Sin embargo, cabe anotar que dicho trabajo no nació sin claros oponentes, la misma autora contaba que una vez hecha su propuesta pudo evidenciar varias caras de escepticismo entre sus colegas, las cuales tuvo que enfrentar hasta que finalmente su proyecto fue posible para fortuna de todos nosotros.

Además de estos que se han llamado “estudios sistemáticos” en ética pragmaticista, se pueden encontrar uno que otro artículo disperso sobre el tema, algunos de ellos no muy alentadores, si bien otros se encuentran más en la línea de la posibilidad de hablar de una ética pragmaticista, como son aquellos de la autoría de Vincent Colapietro, Douglas Anderson y Larry Holmes. Se presenta este panorama con el fin de mostrar algunas circunstancias por las que debe pasar quien decide emprender el trabajo de estudiar sistemáticamente la ética peirceana, a la vez que excusar la modestia o falta de ambición al ofrecer una presentación meramente introductoria.

Ahora bien, es necesario hacer ciertas aclaraciones, porque como diría Aristóteles y tal y como Peirce siempre llevó a cabo sus discusiones filosóficas, no se debe tomar a ningún crítico por tonto y siempre es bueno ponerse en su lugar. Es necesario advertir que no existe un tratado en ética de la autoría de Peirce y que el tema como tal vino a ser tratado por él de manera muy discreta y tardía, si se le compara con otros temas a los que dedicó un amplio número de sus estudios como la lógica, las matemáticas, la geodesia, entre otros. En este sentido, puede resultar bastante extraño querer dar cabida al estudio sistemático de un tema que parece estar olvidado en los anaqueles polvorientos de los intereses peirceanos.

Ante estas críticas es necesario aclarar que, por estudio sistemático se entiende un estudio orgánicamente constituido y abierto a posteriores desarrollos, no un estudio estático, cerrado o finalizado de un problema ya establecido por cierto autor, en este caso, se usarán las palabras de Peirce para definir el campo de estudio de la ética, el interés particular se centra en realizar un estudio sistemático sobre "cuál es el propósito del ser humano" (EP 2.85). Si se entiende un estudio sistemático de esta manera se hace innecesario un tratado sistemático de Peirce sobre la ética, pues no se trata de defender aquí lo que Peirce afirma sobre el tema como si fuera un asunto concluido, sino de establecer una ética siguiendo los pasos de Peirce, es decir, una ética que emerja naturalmente de su proyecto filosófico. Además, cabe anotar, que 
la manera en que Peirce concibe su filosofía no sólo permite sino que promueve este tipo de proyectos. En palabras del autor:

\begin{abstract}
Mi intención es hacer una filosofía a la manera de Aristóteles, es decir, bosquejar una teoría tan comprensiva que, en un largo tiempo por venir, todo el trabajo de la razón humana, en filosofía de cualquier escuela o tipo, en matemáticas, en psicología, en ciencias físicas, en historia, sociología y en cualquier otro departamento del conocimiento que pueda existir, deba aparecer como el llenado de sus detalles. ("A Guess at the Riddle" 1898; CP 1.1)
\end{abstract}

No obstante, esto no quiere decir que Peirce no tenga escritos sobre ética y que realmente la ética tenga un papel meramente tangencial en su filosofía, por el contrario, al pasar de los años y en la medida en que su sistema filosófico crecía y se robustecía, Peirce pudo darse cuenta del papel tan crucial que tienen tanto la ética como la estética en su proyecto filosófico. Digamos entonces que Peirce enfocó sus estudios principalmente en la lógica y que a partir de allí fue interconectando su proyecto con otras áreas del conocimiento, ésta fue la manera en que Peirce encontró los lazos que unen la lógica, la ética y la estética. Esto, además, constituye una particularidad del proyecto peirceano, pues se puede, como muchas veces diría Douglas Anderson en términos coloquiales, "empezar por un lado y terminar en cualquier otro" ya que todo está orgánicamente conectado y estructurado, si bien, valga la pena repetirlo, su sistema no es un sistema cerrado como el de Spinoza, sino más bien evolutivo y abierto al crecimiento.

Igualmente, Peirce es consciente de su finitud, en sus últimos escritos este es un tema recurrente, allí nos habla de su falta de tiempo para resolver un problema y de la necesidad de alguien más que lo escuche para que continúe con su proyecto una vez él ya no esté. Asimismo, Peirce es consciente de su incapacidad para abordar y resolver todos los problemas de la empresa científica y filosófica. Es por ello que en repetidas ocasiones nos recuerda en sus escritos la importancia de la comunidad científica, pues es esta la que debe continuar el trabajo que el individuo ha dejado inacabado. Si bien nuestro destino como individuos es la muerte, nuestras ideas viven en la comunidad que las continúa desarrollando, por consiguiente, es justo decir que más que buscar defensores de su teoría, Peirce buscaba en sus lectores e interlocutores compañeros de viaje que le ayudaran a robustecer su proyecto filosófico desde todos los campos posibles del conocimiento, este proyecto que hoy les quiero presentar es, 
por consiguiente, solamente uno de estos intentos por robustecer la filosofía peirceana, por lo menos en lo que compete al terreno de la ética.

Pero, ¿cómo se explica que Peirce empezara a abordar de manera tan tardía, en el año1902, temas como la ética y la estética? Puede que esto no se deba a un acto premeditado de Peirce, sino a la evolución natural de sus estudios. Como se acaba de señalar, Peirce dedicó la mayor parte de su vida a estudios sobre lógica y desde allí extendió su alcance hacia los estudios en ética y estética. A continuación, se dará una corta versión de cómo fue dicho encuentro.

\section{El encuentro de Peirce con la ética}

Una de las ideas que debe quedar como aprendizaje de esta experiencia es que Peirce nunca pre-estableció las consecuencias a las que le deberían llevar sus estudios, si hizo dichas afirmaciones estas no eran más que hipotéticas, con lo cual se quiere decir que Peirce, como buen aristotélico y científico que era, permitió que la experiencia lo condujera en sus investigaciones y nunca esperó regular la naturaleza a través de su aparataje teórico, como se ve en tantas filosofías que por preservar el orden de sus teorías cierran los ojos ante las sorpresas que el universo les tiene deparadas. Para Peirce, su encuentro con la ética y la estética fue un encuentro al modo científico, donde el fenómeno se vuelve recurrente, sorprende y no queda más que aceptar su existencia y tratar de introducirlo en el aparataje teórico. A continuación se intenta reconstruir dicho encuentro con más detalle.

En sus estudios sobre lógica siempre hubo una idea que rondaba constantemente sus escritos, esta es, la idea del auto-control; es a partir de ésta idea que, Peirce se dio eventualmente al encuentro de la ética y posteriormente de la estética. Existen textos tan tempranos como "la fijación de la creencia", de 1877, donde esto es bastante evidente, tanto así que incluso Peirce lo reconocerá más adelante, en 1906 (es decir, 29 años después), época en la que su interés por la ética y la introducción de esta en su sistema filosófico era ya un hecho. De este modo, afirma en "Temas del pragmaticismo" de 1906 lo siguiente:

Ahora bien, como puede comprobar cualquiera que examine los artículos de noviembre de 1877 [la fijación de la creencia] y de enero de 1878 [Cómo esclarecer nuestras ideas], la teoría del pragmaticismo se basaba, originalmente, 
en un estudio de aquella experiencia de los fenómenos de autocontrol, que es común a todos los hombres y mujeres adultos; $y$, hasta cierto punto, al menos, parece evidente que tiene que haber estado basada siempre así. Pues, el pragmaticismo retrotraería la intención intelectual de los símbolos a las concepciones de la conducta deliberada; y la conducta deliberada es conducta auto-controlada. (EP 2.348)

A continuación se dará un breve recuento del artículo "La fijación de la creencia" de 1877, al que hace aquí referencia Peirce, para esclarecer este punto, pero antes de entrar en la exposición del artículo son necesarios algunos apuntes de contexto. Debe tenerse en cuenta que Peirce concibe la idea de verdad como una idea regulativa a la cual nos acercamos infinitesimalmente a través de la fijación de creencias que tienen como fundamento nuestros aprendizajes a través de la experiencia, esto es lo que él llamará el método científico de fijación de las creencias, por lo cual, dichas creencias son lo mejor que tenemos para acceder a la verdad. Ello no quiere decir, sin embargo, que no logremos desentrañar algo de la verdad, si bien nunca alcanzaremos ese ideal de "verdad", promovido ampliamente en la modernidad, sí nos acercamos a este. Además, cabe señalar que nunca conseguiremos alcanzar la idea de "verdad" al menos por dos razones, primero, porque somos seres finitos y tal idea sería imposible de alcanzar por un solo individuo, como propuso Descartes y, segundo, porque el universo o cosmos se encuentra en constante crecimiento y evolución y la única manera de pensar que la idea de verdad, vendida por la modernidad, sería posible es si el universo fuera estático y regido por leyes necesarias e inmutables.

En su texto de 1877, Peirce se propone como objetivo identificar los diferentes métodos usados por los seres humanos para fijar las creencias. Según Peirce las creencias "guían nuestros deseos y dan forma a nuestras acciones". De este modo, puede decirse que todas nuestras acciones tienen como origen creencias más o menos conscientes, si bien no limitadas meramente al contenido consciente, las cuales se encuentran arraigadas en nuestra alma o mente a modo de hábitos. A este respecto, Peirce clasifica cuatro métodos de fijación de la creencia, a saber, el método de la tenacidad, el método por autoridad, el método a priori y, finalmente, el método de la ciencia.

El método de la tenacidad consiste en aferrarse a una creencia determinada, en palabras de Peirce: "reiterándonosla constantemente a nosotros mismos, deteniéndonos en todo lo que puede conducir a tal creencia, y aprendiendo a alejarnos con desprecio 
y aversión de todo lo que pueda perturbarla", según Peirce, muchos hombres se adhieren a este método de fijación de la creencia, que, no obstante, se ve minado cuando nos damos cuenta de que muchas personas piensan de modo diferente y que la experiencia lo puede contradecir con facilidad.

El método de la autoridad lo describe de la siguiente forma:

Dejemos, pues, actuar la voluntad del estado en lugar de la del individuo. Que se cree una institución que tenga por objeto mantener correctas las doctrinas ante la gente, reiterarlas perpetuamente, y enseñarlas a los jóvenes; teniendo a la vez poder para evitar que se enseñen, defiendan, o expresen, doctrinas contrarias. Que se alejen de la perspectiva de los individuos todas las causas posibles de un cambio mental. Mantengámosles ignorantes, no sea cosa que por alguna razón aprendan a pensar de modo distinto a como lo hacen. Asegurémonos de sus pasiones, de manera que vean con horror y hostilidad las opiniones privadas y poco usuales. Reduzcamos entonces al silencio a todos los que rechacen la creencia establecida. Que la gente los eche y los embadurne cubriéndolos de plumas, o que se investigue el modo de pensar de las personas sospechosas, y que si se las encuentra culpables de creencias prohibidas se las someta a algún castigo ejemplar. Cuando en todo caso no se pueda conseguir una total anuencia, una masacre general de todos los que no piensen de una determinada manera se ha acreditado como un medio muy efectivo de establecer opinión en un país. Si se carece de poder para hacerlo, redactemos una lista de opiniones a la que nadie con la más mínima independencia de criterio pueda asentir, y exijamos que los fieles acepten todas estas proposiciones con objeto de aislarlos lo más radicalmente posible de la influencia del resto del mundo. (CP 5.379; EP 1.117)

En tercer lugar, está el método a priori, según Peirce este es el método propio de la filosofía metafísica, sobre el cual afirma:

Usualmente los sistemas de este tipo [es decir, los sistemas metafísicos] no se han basado en hechos observados, al menos no a un cierto nivel relevante. Básicamente se han adoptado porque sus proposiciones fundamentales parecían “agradables a la razón”. Es esta una expresión adecuada; no significa aquello que concuerda con la experiencia, sino aquello que nos encontramos inclinados a creer. (CP 5.382; EP 1.118-119) 
Finalmente, encontramos el método científico. Según Peirce, este es el único método que fija sus creencias teniendo en cuenta la experiencia o "la realidad" y el cual él desarrollará posteriormente con su teoría lógica de la abducción, como fuente de las hipótesis creativas; la inducción, como el método para recoger datos experimentales con el fin de corroborar o desaprobar nuestras hipótesis; y la deducción como el modo de corroborar o refutar las hipótesis con miras en sus posibles implicaciones. Peirce se considera a sí mismo realista y criticará fuertemente al nominalismo en otros de sus textos. Discusión que se puede observar en textos como "Fraser's The Works of George Berkley", entre otros.

Algunas virtudes que encuentra Peirce en el método científico y que me gustaría resaltar son las siguientes: una es que "el impulso social no nos lleva a ponerlo en duda", ya que todos más o menos compartimos una idea de realidad y cuando hay divergencias el método nos permite entrar en discusiones y experimentos para dirimir el problema, no como en el método de tenacidad que lleva finalmente al ostracismo. Además, afirma Peirce que: [é]ste es el único de los cuatro métodos que presenta una cierta distinción entre una vía recta y otra errónea”, pues todos los demás cierran sus ojos ante la experiencia.

Una de las consecuencias más significativas de este texto, como Larry Holmes apunta, pero no es muy tenido en cuenta por los intérpretes en general, es que "la adopción del método científico es una elección y esta es normativa de una manera vitalmente importante. El valor de éste [...] método particular [es decir, el método científico] es que es controlado y se auto-corrige" $(1966,114)$. En otras palabras, la manera en que nos acercamos al conocimiento del universo está basada fundamentalmente en una elección de tipo ético. Resulta innegable entonces que la idea de auto-control haya ejercido desde sus inicios una fuerte influencia en el pensamiento de Peirce, la cual fue tomando cada vez mayor fuerza y vivacidad en su concepción del cosmos.

Existe otra reflexión de Peirce que parece dar cuenta exactamente del modo en que la idea de auto-control se introdujo es sus preocupaciones filosóficas y fue creciendo hasta el punto de enfrentarlo con el vasto mundo de la ética. En su artículo de 1892 titulado "La ley de la mente" Peirce establece la ley de la mente del siguiente modo:

El análisis lógico aplicado a los fenómenos mentales muestra que no hay más que una ley de la mente, a saber, la de que las ideas tienden a propagarse de forma continua y a afectar a algunas otras que se encuentran en una relación 
peculiar de afectabilidad respecto de aquéllas. Al propagarse pierden intensidad, y, especialmente, el poder de afectar a otras, pero ganan en generalidad y acaban por mezclarse con otras ideas. (EP 1.313)

Por consiguiente, si bien el interés principal de Peirce se desarrolló alrededor de la lógica por muchos años, él se encontró la idea de auto-control en una primera instancia de modo más o menos incipiente hasta el momento en que esta idea cogió cada vez más vida y logró afectar tanto el pensamiento de Peirce como su sistema filosófico en general.

De este modo, en la medida en que su idea de la lógica fue creciendo y robusteciéndose, incluso su idea respecto al papel de la lógica se vio afectada. En uno de sus textos maduros de 1906 "las bases del pragmaticismo en las ciencias normativas" Peirce reconoce, por ejemplo, que en un principio concibió la lógica como "la ciencia de las leyes formales de la relación de los símbolos con sus objetos” (EP2. 387) pero que posteriormente se pudo dar cuenta de que ésta no debía estar limitada a los símbolos sino que debía comprender el estudio de todo tipo de signos (Cf. EP2. 387). Asimismo, eventualmente pudo darse cuenta de que la lógica no solamente era el estudio del razonamiento sino que el razonamiento mismo es pensamiento auto-controlado y de que, en esta medida, existe un paralelismo entre la conducta auto-controlada y el razonamiento, sobre esto afirma Peirce, "el razonamiento es esencialmente pensamiento bajo auto-control, del mismo modo en que la conducta moral es conducta bajo auto-control. De hecho, el razonamiento es una especie de conducta controlada y como tal necesariamente comparte los rasgos esenciales de la conducta controlada" (EP 2.249). No resulta extraño entonces que desde comienzos del siglo XX, Peirce recurra en sus escritos a dar ejemplos de carácter ético para ejemplificar procesos de razonamiento lógico. Pero la relación entre lógica y ética no se quedará en este simple paralelismo. Peirce finalmente descubrirá que la ética es una ciencia normativa para la lógica.

Peirce entiende las ciencias normativas como las ciencias que estudian lo que debería ser, estas son, estética, ética y lógica. Dichas ciencias están establecidas según el modelo de sus tres categorías, a saber, la estética está relacionada con el sentimiento y corresponde a la categoría de primeridad, la ética está relacionada con la acción o la conducta y corresponde a la categoría de segundidad, finalmente, la lógica está relacionada con el pensamiento y la mediación, que corresponde a la categoría de terceridad. 
Diagrama 1. Las ciencias normativas

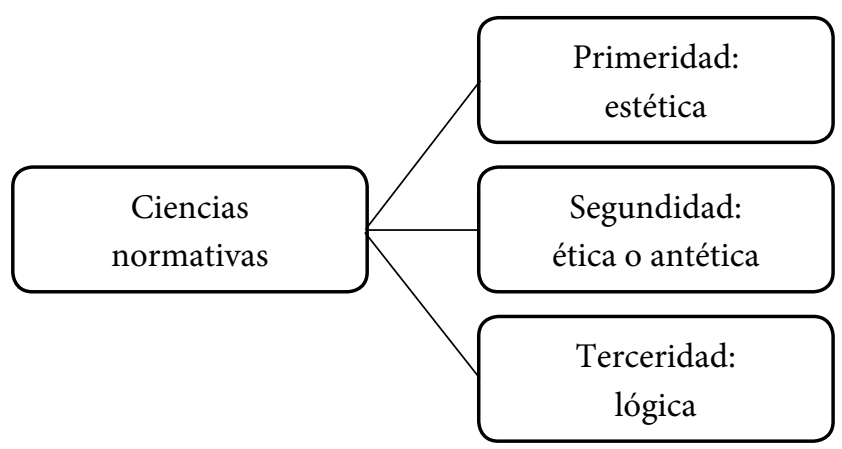

Para muchos, decir que la estética es una ciencia normativa para la ética y esta última para la lógica suena bastante extraño, sobre todo para aquellos que no están familiarizados con el ideal de kaloskagathía de los antiguos, según el cual, el bien y la belleza constituyen un ideal de belleza y armonía, no sólo en el cosmos sino también para el ser humano. Dicha extrañeza también es debida, en parte, a la visión mecanicista del universo que nos heredaron los modernos y a la idea más o menos dispersa de que cuando hablamos de ciencias normativas estas implican "condiciones de posibilidad" al modo kantiano. Siendo así, la idea de Peirce se acerca más al ideal de los antiguos, si bien tiene sus propias particularidades.

Primero, se debe tener claro que cuando Peirce llama a sus ciencias normativas, estética y ética con ello no se debe entender a la estética como la teoría del gusto y a la ética como a la teoría de la conducta correcta conforme a las convenciones sociales. De hecho, con el fin de evitar dicha confusión Peirce quiso cambiar el nombre de ética por el de antética en 1906. Como él mismo lo afirma:

[n]o hay duda de que ellas [las ciencias normativas] están íntimamente relacionadas con los tres tipos de artes correspondientes, o ciencias prácticas [es decir, con la teoría del gusto y la conducta humana]. Pero aquello que hace la palabra normativa necesaria (y no puramente ornamental) es precisamente el hecho singular de que, pese a que estas ciencias estudian lo que debe ser, esto es, los ideales, ellas son las más puramente teoréticas de las ciencias más puramente teoréticas. (CP 1.281) 
En consecuencia, como afirmará años más tarde, el que las ciencias normativas sean ciencias puramente teoréticas no impide que puedan ser un posible determinante de la conducta (EP 2.372). De este modo, cuando se habla aquí de ciencias normativas, se debe evitar pensar que éstas son algo así como una "condición de posibilidad o fundamento" para algo más, en un sentido mecanicista, es decir, que va de causa a efecto, por el contrario, teniendo en cuenta que Peirce está defendiendo un modelo teleológico y evolucionista se debe pensar que las ciencias normativas condicionan o tienen efecto, principalmente, en la acción futura, esto es, en los posibles hábitos de conducta que puedan determinar.

Ahora bien, volviendo a la relación entre ética y lógica, afirma Peirce:

la lógica no es un estudio integral del razonamiento, sino solamente sobre si las condiciones del razonamiento son buenas o malas, $y$ si buenas en qué medida y en qué aplicación. Ahora, el buen razonamiento es el razonamiento que consigue su propósito. Su propósito es proveer una guía para la conducta,- - y el pensamiento, al ser una operación activa, es una especie de conducta,-en caso de que ningún percepto [esto es la primera información a la que accedemos por medio de la percepción] del cual un juicio pudiera estar directamente formado, esté a la mano. Su objeto, es decir, lo que el razonador pensará bien cuando ese percepto ocurra, o qué pensaría si ocurriese. (EP 2.386-387)

Por consiguiente, dirá Peirce, que para establecer qué es un razonamiento correcto o incorrecto se tiene que tener un fin o ideal en mente y este ideal es el que ha de proveer el estudio de la ética o antética. La antética, entonces, tiene como objetivo establecer la naturaleza del summum bonum o idea del bien último, así como la teoría de la conformidad de la acción con dicho ideal (Cf. EP 2.377). Este ideal, a su vez, debe ser establecido primero por la estética en tanto que esta es la ciencia que determina lo que es admirable per se y sin miras a posteriores justificaciones. Para resumir, en primer lugar, la estética es normativa para la ética o antética, la cual tiene por fin el estudio de lo admirable per se, esto es, el ideal entendido desde el punto de vista del sentimiento. En segundo lugar, la antética es normativa para la lógica al proveer la idea del summum bonum, el cual constituye un aspecto del admirable per se y que estaría dado paulatinamente en virtud de la experiencia como especie en este mundo y que, además, como el mismo Peirce sostiene, no puede constituir un ideal cerrado ni único, sino la idea más vaga y general de todas, la cual se encuentra en constante 
crecimiento y desarrollo. Finalmente, los medios o razonamientos correctos que nos permitirían alcanzar ese summum bonum son determinados por la lógica.

\section{El summum bonum}

Para finalizar, se dará una corta caracterización del summum bonum de modo que quede más clara la idea que tiene Peirce en mente sobre el lugar de la ética en su proyecto filosófico. De manera sucinta puede decirse que el summum bonum es la ley, ideal o causa final que rige el crecimiento del universo a través del auto-control, la cual constituye, según Peirce, la idea de razonabilidad en sí misma (Cf. EP 2.343-344). En otras palabras, para Peirce, tal y como lo explica David Pfeifer, en la medida en que el universo y con él, la idea del summum bonum se desarrolla, en palabras del autor:

las cosas se vuelven más regulares, más armoniosas. Pero parece que las nuevas cosas están convirtiéndose en parte del universo; el universo se está haciendo más complejo. Estos dos procesos son el resultado de la evolución del universo; ellos también caen bajo el alcance del summum bonum. El summum bonum finalmente afectará a todas las cosas. Todas las cosas, incluso aquellas que no se han actualizado aún, serán armonizadas por su acción. (Pfeifer, 1971, p. 12)

Visto de este modo, el summum bonum pareciera más un acto del destino que un acto creativo del que el hombre pudiera participar y, en cierta medida, es así. No obstante, el summum bonum constituye principalmente un acto creativo en el que el ser humano, como el resto de las creaturas, tienen cierto grado de participación según su capacidad de auto-control y de imaginar o prever el futuro. Además, debe tenerse en cuenta que, para Peirce, si se quiere ser racional y con ello, que el ideal de felicidad armonice y se encarne en el universo, no sólo se deben unir los intereses con la comunidad humana, sino también con la comunidad universal, es decir, se debe desarrollar un ideal de felicidad o un summum bonum particular que esté, no sólo en conexión con la comunidad humana en el sentido más amplio posible, sino también que ayude al cosmos a alcanzar la armonía entre sus creaturas y ello sólo es posible a través de la creatividad. No debe ser ajena la idea de que para conciliar muchos puntos de vista frente a un objetivo común sin ejercer violencia, creatividad y simpatía, punto que no se alcanza a abordar en el momento, pero que Peirce desarrolló en muchos de sus escritos, deben estar a la orden del día. 
Recordemos que Peirce piensa que las ideas tienden a propagarse y afectar a otras, se mezclan y alcanzan generalización, este constituye el proceso de crecimiento de las ideas y la ley de la mente anteriormente expuesta. Pero la ley de la mente no acaba aquí, Peirce también piensa que las ideas vivas se encarnan en el mundo, porque lo afectan y le dan forma al universo, en este sentido, Peirce debe ser considerado como cierto tipo de idealista. Él, por ejemplo, considera que las ideas de "justicia” y "verdad" son las ideas más poderosas que mueven el mundo (EP 2.343) y, ¿cómo no darle la razón?, ¿ cómo explicar entonces que las mujeres y aquellos que anteriormente considerábamos como esclavos hayan podido acceder al voto, sino como una muestra de que la idea de justicia le puede dar forma al mundo y lo continúa haciendo? Dejar estas cosas al azar, como algunos han pretendido, es más que menospreciar el esfuerzo y la lucha de muchos seres humanos por alcanzar estos objetivos.

De esta forma, según Peirce, nosotros podemos ayudar a la realización del summum bonum en el universo, pese a nuestra finita y pequeña existencia, pues "no es tratando con fría justicia el círculo de mis ideas que puedo hacerlas crecer, sino al acariciarlas y cuidarlas como lo haría con las flores de mi jardín” (CP 6.289). Es pues, deber ético, si se quiere, simpatizar con el universo y ayudarlo a crecer armónicamente en la medida de las capacidades humanas, pero también con la esperanza de que otros continuarán el trabajo siempre inacabado.

Algunas conclusiones sobre lo anteriormente dicho, primero, sí es posible, justo y necesario realizar estudios en el área de la ética conforme a lo expuesto sobre el proyecto filosófico del pragmaticismo periceano. No sólo porque el mismo Peirce invita a ello, sino también porque las herramientas para emprender este estudio ya han sido propuestas por el mismo Peirce. Segundo, conforme a lo dicho acerca de los planteamientos éticos de Peirce sobre las ideas de auto-control, las ciencias normativas y el summum bonum, el ser humano tiene el deber ético de pensar, esto es, de usar la lógica, en el amplio sentido de la palabra, para crear su propia idea de felicidad o su summum bonum y procurar que este ideal no solamente se una con los propósitos del universo, sino que procure mejorarlo conforme a sus posibilidades. 


\section{Referencias}

Anderson, D. R. (1999). Peirce: Ethics and the Conduct of Life. In Classical American Pragmatism: Its Contemporary Vitality, Urbana-Champaign: University of Illinois Press.

Boero, H. (2014). Charles S. Peirce: Claves para una ética pragmaticista. Barañáin: EUNSA.

Colapietro, V. (Summer 2009). Habit, Competence, and Purpose: How to Make the Grades of Clarity Clearer. Transactions Of The Charles S. Peirce Society 45, no. 3: 348-377.

Holmes, L. (1966). Peirce on Self-Contro, Transactions of the Charles S. Peirce Society, II/2, 113-130.

Misak, C. (2004). C. S. Peirce on Vital Matters, en C. Misak (ed.), The Cambridge Companion to Peirce, Cambridge University Press, Cambridge, 150-174.

. (2004). Truth and the end of inquiry a Peircean account of truth (Expanded pbk. ed.). Oxford: Clarendon Press

. (2002).Truth, politics, morality pragmatism and deliberation. London: Routledge

Peirce, C. S. Collected Papers of Charles Sanders Peirce, vols. 1-8, C. Hartshorne, P. Weiss y A. W. Burks (eds.), Harvard University Press, Cambridge, MA, 1931-1958. Edición electrónica de J. Deely, InteLex, Charlottesville, VA, 1994. (1992-1998). (EP) The Essential Peirce. Selected Philosophical Writings, vols.

1-2, N. (s.f.) Houser et al. (eds.), Indiana University Press, Bloomington.

Petry, E. Jr., (1992). The Origin and Development of Peirce's Concept of Self-Control, Transactions of the Charles S. Peirce Society, XXVIII/4, 667-690.

. (1990). Self-control in the philosophy of Charles S. Peirce, Doctoral Dissertation. Pennsylvania State University, PA.

Pfeifer, D. (1971). The Summum Bonum in the Philosophy of C. S. Peirce, Doctoral Dissertation, University of Illinois, Urbana, IL. 